\title{
INFLUENCIA DE LA ANSIEDAD Y SÍNTOMAS DEPRESIVOS SOBRE LA AGENCIA DE AUTOCUIDADO EN CUIDADORES DE PERSONAS CON ENFERMEDAD CRÓNICA
}

\author{
INFLUENCE OF ANXIETY AND DEPRESSIVE SYMPTOMS ON SELF-CARE AGENCY IN CAREGIVERS OF PEOPLE \\ WITH CHRONIC DISEASE
}

Isaí A. Medina, Ana L. Carrillo, Martin Pantoja, Reyna Torres, Josué A. Medina y Luis C. Cortez

Universidad Autónoma de Coahuila (México)

\begin{abstract}
El aumento de enfermedades crónicas y de sus complicaciones ha llevado a la necesidad de tener un cuidador, sin embargo, el ser cuidador primario puede ocasionar un impacto en la salud mental, siendo un factor predisponente para desarrollar estados afectivos negativos, como son la ansiedad y síntomas depresivos y que pueden tener una repercusión en el autocuidado. Objetivo. Determinar la influencia de la ansiedad y síntomas depresivos sobre la capacidad de agencia de autocuidado en cuidadores informales de personas con enfermedad crónica. Metodología. Se aplicó una cédula de datos sociodemográficos, la subescala de ansiedad estado (IDARE), CES-D para síntomas depresivos y escala de apreciación de autocuidado. El análisis de datos fue con SPSS versión 22, con estadística descriptiva e inferencial. Resultados. La muestra estuvo constituida por 94 participantes, la ansiedad obtuvo una $M=46.8(D E=8.93)$, para los síntomas depresivos fue de $M=27.13$, ( $D E=13.21)$ y para la capacidad de agencia de autocuidado fue de $M=63.82$ ( $D E=6.34)$, ambas variables son predictoras $(p<.05)$ e influye en un $15 \%$ sobre la capacidad de agencia de autocuidado. Conclusión. Estos resultados servirán para establecer bases de intervenciones multidisciplinarias que mejoren la calidad de vida y bienestar en los cuidadores.
\end{abstract}

Palabras clave: enfermedad crónica, autocuidado, ansiedad, depresión.

\begin{abstract}
The increase in chronic diseases and their complications has led to the need for a caregiver. However, caring for a long period can have an impact on mental health, which is why it is a predisposing factor to develop negative affective states, such as anxiety and depression, which can have an impact on selfcare behavior. Objective. Identify the influence of anxiety and depressive on the capacity of self-care agency in informal caregivers of people with chronic disease. Methodology. A sociodemographic data card, the state anxiety scale (IDARE), CES-D for depressive symptoms, and self-care assessment scale were administered. For the data analysis, it was with SPSS version 22, with descriptive and inferential statistics. Results. The sample consisted of 94 participants, the median of state anxiety was $M=46.8$ (SD $=8.93)$, for depressive symptoms it was $M=27.13,(S D=13.21)$ and for self-care agency capacity it was $M=63.82(S D=6.34)$, it was shown that both variables are predictors $(p<.05)$ and influence $15 \%$ on the capacity of self-care agency. Conclusions. These results will serve to establish bases for multidisciplinary interventions that improve the quality of life and well-being of caregivers.
\end{abstract}

Isaí Arturo Medina Fernández, Universidad Autónoma de Coahuila, México, https://orcid.org/0000-0003-2845-4648 Ana Laura Carrillo Cervantes, Universidad Autónoma de Coahuila, México, correspondencia a anacarrillo@uadec.edu.mx, https://orcid.org/0000-0003-2920-4675

Martin Pantoja Herrera, Instituto Nacional de Rehabilitación “Luis Guillermo Ibarra Ibarra”, https://orcid.org/0000-0002-8171-6865

Reyna Torres Obregón, Universidad Autónoma de Coahuila, México. Josué Arturo Medina Fernández, Universidad Autónoma de Coahuila, México, https://orcid.org/0000-0003-3546-6970

Luis Carlos Cortez González, Universidad Autónoma de Coahuila, México, https://orcid.org/0000-0002-1655-0272 
Keywords: chronic disease, selfcare, anxiety, depression.

Los cambios sociales, culturales y de salud ha ocasionado un incremento a nivel mundial de enfermedades crónicas; este cambio trae consigo complicaciones que podría afectar en los diferentes niveles de dependencia, aumentando la necesidad de un cuidador informal, lo que implica el asumir las tareas de la vida diaria para disminuir el impacto negativo que tiene la dependencia sobre la calidad de vida y bienestar de la persona a quien cuida (Torres-Avendaño et al., 2018).

Dentro de las enfermedades crónicas, destacan las incapacitantes, las cuales son consideradas aquellas que provocan el deterioro físico y/o cognitivo progresivo de la persona que la sufre, convirtiéndose progresivamente en una persona con una dependencia en las actividades básicas y/o de la vida diaria para poder realizar su autocuidado, requiriendo de un cuidador (Mercado et al., 2018).

En este sentido, el cuidador es el recurso, instrumento y medio por el cual se proveen cuidados específicos y muchas veces especializados a los enfermos crónicos, es decir, en ellos se deposita o descansa el compromiso de preservar la vida de otro. Este hecho tiene una fuerte repercusión en la calidad de vida del cuidador, afectando no solo la esfera física y psicológica, sino también la social y económico (Cruz-Rivas \& Ostiguin, 2011; Mercado et al., 2018).

La esfera psicológica se ve repercutida en el cuidador informal, debido a que son más propensos a desarrollar ansiedad y depresión, estas dos variables, crea en la persona un hábito de preocupación, cambios de humor y temor constante que lo mantiene con una tensión más allá de lo normal y que afectan la calidad de vida del cuidador (Blázquez et al., 2012; García, 2018; Ortiz et al., 2018).

Estos dos estados afectivos negativos son más prevalecientes en esta población debido a que aumenta la vulnerabilidad, derivadas de la sobrecarga que se asocia con afecciones físicas, descompensación de enfermedades padecidas, falta de adherencia al tratamiento e ineficientes habilidades de autocuidado (Martínez, 2018).

Relacionado con lo anterior, entre las tareas del cuidador se encuentra las responsabilidades del cuidado mismo, participar en la toma de decisiones conjuntas y brindar cuidados a su paciente (Cruz-Rivas \& Ostiguin, 2011), sin embargo, el primer punto se ve afectado por enfocar toda su energía en las demás actividades que satisfagan las necesidades de la otra persona, limitando la agencia de autocuidado y pudiendo traer consecuencias a su salud.

La capacidad de agencia de autocuidado es una cualidad, aptitud o habilidad como capacidad fundamental que agrupa las habilidades básicas del individuo (sensación, percepción, memoria y orientación), los componentes de poder (capacidades específicas relacionadas con la habilidad del individuo para comprometerse en el autocuidado) y las capacidades para operaciones de autocuidado (Gast et al., 1989). Esta variable se va vulnerando en el cuidador, ya que vela por el bienestar de su paciente, olvidándose su bienestar propio o conducta de autocuidado (Hernández et al., 2014).

Según la evidencia científica, en los estudios realizados en América latina han demostrado una relación de diversos factores como la ansiedad y depresión con la capacidad de agencia de autocuidado ( $p<.05)$ (Haro, 2014; Méndez et al., 2010; Paz-Rodríguez, 2010). Estos estados 
afectivos negativos son ocasionados por un estrés crónico debido la acción de cuidar a una persona con condición crónica, el cual es un estado de amenaza hacia la salud mental del cuidador, pudiendo ser un factor que ocasione una dificultad persistente de autocuidado debido a los problemas psicológicos y emocionales, todo ello, tiene como repercusión una sobrecarga del cuidador o daños a la salud (Retamal-Matus et al., 2015).

Así mismo, el desplazamiento de cuidados cada vez más complejos en el paciente con condición crónica, ocasiona la búsqueda de atención más especializada como en el tercer nivel de atención. Lo anterior no solo lo vuelve compleja en el área clínica, si no una atención más enfocada por el sistema de cuidados informales (López et al, 2016). Esta mayor demanda de cuidador puede aumentar el impacto en la esfera psicológica y disminución en el autocuidado

Dada la importancia actual de estudiar al cuidador informal de personas con enfermedad crónica, es necesario plantear bases para la identificación de factores, como los es la esfera psicológica, que pueden influir en el autocuidado, con ello se puede plantear futuras intervenciones que disminuyan el riesgo de afecciones físicas y emocionales. Referido lo anterior surge la necesidad de plantear el siguiente objetivo: determinar la influencia de ansiedad y síntomas depresivos sobre la capacidad de agencia de autocuidado en cuidadores infórmales de personas con enfermedad crónica.

\section{Método}

\section{Diseño}

El diseño de esta investigación es correlacional predictivo, transversal, debido a que se analiza la predicción de las relaciones causales entre las variables y transversal por que sucede en un mismo periodo de tiempo (Grove \& Gray, 2019).

\section{Participantes}

La muestra estuvo confirmada por 94 cuidadores informales de pacientes con condición crónica que consultaban en un hospital de tercer nivel de atención. El muestreo fue de tipo no probabilístico por conveniencia, se contemplaron como criterios de inclusión: ser cuidador informal de paciente con alguna condición crónica, ser cuidador con un mínimo de 2 meses, ser mayor de edad (mayor o igual a 18 años) y que su familiar quien cuida consulte en el hospital de tercer nivel de atención de la Ciudad de México.

\section{Instrumentos}

Cédula de datos sociodemográficos en el que se recabó información como sexo, edad, nivel de estudio, ocupación, años de cuidador, actividades que desarrolla como cuidador, enfermedades crónicas o afecciones físicas padecidas, e ingreso mensual en pesos mexicanos.

Escala de apreciación de autocuidado. Elaborado por Evers, Isenberg, en idioma español versión para la población mexicana de la Dra. Esther Gallegos con alfa de Cronbach de .77, el instrumento está constituido por un total de 24 ítems que mide la capacidad de agencia de autocuidado y se responde mediante una escala tipo Likert. El rango de puntuación 24 a 120; a mayor puntuación, mayor capacidad de agencia de autocuidado (Gallegos, 1998). 
Inventario de Ansiedad, subescala ansiedad estado (IDARE) con un Alpha de Cronbach de .80, fue desarrollado para medir el grado de malestar que resulta de estímulos ambientales y que son vistos por los individuos como amenazas, está constituido por 20 reactivos y se responde con una escala tipo Likert, cuya puntuación oscila entre 29 a 80, es decir a mayor puntaje mayor ansiedad percibida (Spielberger \& Díaz-Guerrero, 2002).

EI CES-D proporciona indicadores de riesgo de depresión, a través de la expresión de síntomas depresivos, cuenta con un Alpha de Cronbach de .88. El instrumento se responde con escala tipo Likert donde, la puntuación oscila de 0 a 60 puntos, donde a mayor puntuación mayor presencia de síntomas depresivos (Bojórquez \& Salgado, 2009; Haro, 2014).

\section{Procedimiento}

Los participantes fueron reclutados en la Ciudad de México en un Hospital de tercer nivel de atención, entre el mes de junio y julio 2019. A todos los participantes voluntarios se les informó el objetivo del estudio y el proceso de participación que fue mediante la administración de lápiz y papel. Aceptado la participación, se procede a leer el consentimiento informado, posteriormente se aplicó una cédula de datos, esta fue elaborada por los autores y posee preguntas que permitieron caracterizar a la población, seguido se cuestionó sobre la capacidad de agencia de autocuidado, y por último dos instrumentos que determinaron los estados afectivos negativos en el cuidado, como la ansiedad estado y síntomas depresivos

El presente estudio fue aprobado por el comité de ética e investigación de la Facultad de Enfermería de la Universidad Autónoma de Coahuila y se apegó a lo dispuesto en la Ley General de Salud en materia de Investigación para la Salud, en el titulo segundo de los aspectos éticos de la investigación en seres humanos, respetándose la dignidad y la protección de sus derechos y bienestar, además de la protección de la privacidad y se aplicó un consentimiento informado $(\mathrm{H}$. Congreso de la Unión, 2012).

\section{Análisis de los datos}

El análisis estadístico fue a través del programa Stadistic Package for the Social Science (SPSS) de Windows versión 22 a través de estadística descriptiva para variables nominales y ordinales, posteriormente se determinó prueba de normalidad para variables cuantitativas, obteniendo una distribución no normal ( $p$ <.05). La correlación fue mediante el coeficiente de correlación de Spearman, diferencia de medias entre 2 grupos con Kruskall Wallis y para más de 2 grupos con $U$ Mann Whitney; se utilizó los estadísticos de regresión lineal simple para el objetivo general.

\section{Resultados}

La muestra estuvo constituida por 94 participantes con una edad ( $M=45.83, \mathrm{DE}=14.92$ años), siendo en su mayoría mujeres, amas de casa, casados, cuidando a un hijo, cuyas funciones más prevalentes fue el apoyo en las actividades de la vida diaria (ver Tabla 1).

Tabla 1

Características de los cuidadores informales

\begin{tabular}{lll}
\hline Variable & $\mathrm{Fr}$ & $\%$ \\
\hline Sexo & & \\
\hline
\end{tabular}




\begin{tabular}{|c|c|c|}
\hline Hombre & 29 & 30.9 \\
\hline Mujer & 65 & 69.1 \\
\hline \multicolumn{3}{|l|}{ Ocupación } \\
\hline Empleado & 25 & 26.6 \\
\hline Ama de casa & 47 & 50 \\
\hline Profesional & 6 & 6.4 \\
\hline Estudiante & 10 & 10.6 \\
\hline Jubilado & 6 & 6.4 \\
\hline \multicolumn{3}{|l|}{ Estado civil } \\
\hline Soltero & 23 & 24.5 \\
\hline Casado & 49 & 52.1 \\
\hline Divorciado & 6 & 6.4 \\
\hline Unión libre & 12 & 12.8 \\
\hline Viudo & 4 & 4.3 \\
\hline \multicolumn{3}{|c|}{ Parentesco de la persona que cuida } \\
\hline Hijo & 40 & 42.6 \\
\hline Esposa/esposo & 34 & 36.2 \\
\hline Nieto/nieta & 5 & 5.3 \\
\hline sobrino & 2 & 2.1 \\
\hline hermano & 4 & 4.3 \\
\hline conocido & 3 & 3.2 \\
\hline abuelo & 6 & 6.5 \\
\hline \multicolumn{3}{|l|}{ Afecciones físicas } \\
\hline Diabetes & 12 & 12.8 \\
\hline Hipertensión arterial & 12 & 12.8 \\
\hline Problemas de la columna & 12 & 12.8 \\
\hline Problemas en las rodillas & 15 & 16 \\
\hline Problemas en los pies & 5 & 5.3 \\
\hline Padece alteraciones del sueño & 9 & 9.6 \\
\hline \multicolumn{3}{|c|}{ Actividades que realiza a su familiar } \\
\hline Acompañamiento & 21 & 22.3 \\
\hline Apoyo en las AIVD & 32 & 33.0 \\
\hline Apoyo en las ABVD & 26 & 27.7 \\
\hline Cuidador de tiempo completo & 16 & 17.0 \\
\hline
\end{tabular}

Nota: $\mathrm{Fr}=$ Frecuencia, \%= Porcentaje, $\mathrm{AIVD}=$ Actividades instrumentales de la vida diaria, $\mathrm{ABVD}=$ Actividades instrumentales de la vida diaria, $\mathrm{N}=94$.

La escolaridad, oscilo entre los 0 y 23 años con una $M=12.01$ ( $D E=4.18$ ), así mismo el tiempo del cuidado se encontró entre los 2 y 480 meses con una $M=83.68$ ( $D E=90.32)$ meses y el ingreso mensual familiar estuvo entre $\$ 1200$ y $\$ 18000$ mensuales con una $M=5176.81$ ( $D E=3408.85$ ) pesos mexicanos.

La ansiedad de los cuidadores obtuvo una $M=46.84$ ( $D E=9.83$ ); al determinar diferencia de medias entre las características del cuidado, se identificó que la ansiedad fue diferentemente 
significativa en la ocupación del cuidador y actividades que realiza el cuidado, siendo mayor en mujeres que trabajan en el hogar y que realizan apoyo en las actividades instrumentales de la vida diaria (AIVD) (Tabla 2).

Tabla 2

Diferencia de medias de acuerdo al sexo, ocupación, estado civil y actividades que realiza el cuidador

\begin{tabular}{|c|c|c|c|c|c|c|}
\hline \multirow[t]{2}{*}{ Variable } & \multicolumn{2}{|c|}{ Ansiedad estado } & \multicolumn{2}{|c|}{ Síntomas depresivos } & \multicolumn{2}{|c|}{$\begin{array}{l}\text { Capacidad de } \\
\text { agencia de } \\
\text { autocuidado }\end{array}$} \\
\hline & $M(D E)$ & $p$ & $M(D E)$ & $p$ & $M(D E)$ & $p$ \\
\hline Sexo & & -.507 & & .990 & & .892 \\
\hline Mujer & $46.35(9.45)$ & & 27.01(12.77) & & $63.84(6.55)$ & \\
\hline Hombre & 47.93(10.71) & & 27.41(14.38) & & $63.79(5.91)$ & \\
\hline Ocupación & & .001 & & .002 & & .001 \\
\hline Empleado & $43.63(8.80)$ & & 27.92(12.22) & & $60.76(6.28)$ & \\
\hline Trabajo del hogar & 48.14(11.10) & & $30.68(13.90)$ & & $64.04(6.06)$ & \\
\hline Profesional & 46.86(7.70) & & $15.66(6.62)$ & & $68.50(3.14)$ & \\
\hline Estudiante & 43.10(6.74) & & $16.20(6.84)$ & & $69.40(3.92)$ & \\
\hline Jubilado & $44.83(9.94)$ & & $25.83(10.22)$ & & $61.00(6.26)$ & \\
\hline Estado civil & & .393 & & .023 & & .090 \\
\hline Soltero & 43.52(6.84) & & 20.43(12.34) & & $65.26(7.78)$ & \\
\hline Casado & 48.67(10.98) & & $30.53(12.65)$ & & $63.95(5.75)$ & \\
\hline Divorciado & $43.66(8.26)$ & & 25.50(10.67) & & $57.66(4.76)$ & \\
\hline Unión libre & $47.58(6.97)$ & & $26.16(12.20)$ & & $63.50(6.27)$ & \\
\hline Viudo & $46.00(16.35)$ & & $29.50(5.25)$ & & $64.25(1.25)$ & \\
\hline $\begin{array}{l}\text { Actividades que } \\
\text { realiza el cuidador }\end{array}$ & & .044 & & .035 & & .129 \\
\hline Acompañamiento & 45.42(9.17) & & 26.19(13.39) & & $62.80(5.79)$ & \\
\hline Apoyo en las AIVD & $51.29(10.99)$ & & $32.70(13.88)$ & & $62.29(6.77)$ & \\
\hline Apoyo en las ABVD & $45.34(8.86)$ & & $22.53(13.26)$ & & $65.50(6.62)$ & \\
\hline $\begin{array}{l}\text { Cuidador de tiempo } \\
\text { completo }\end{array}$ & $42.50(6.80)$ & & $25.06(7.61)$ & & $65.43(5.12)$ & \\
\hline
\end{tabular}

Así mismo, los síntomas depresivos obtuvieron una $\mathrm{M}=27.13$ ( $\mathrm{DE}=13.21$ ), encontrándose una diferencia significativa de depresión según la ocupación, estado civil y actividades que realiza el cuidador, siendo mayor en las trabajadoras del hogar, casados y quien apoya en las AIVD. Por último, la capacidad de agencia de autocuidado tuvo una $M=63.82$ ( $D E=6.34$ ), hallándose una diferencia significativa en la ocupación, siendo menor en los empleados (Tabla 2).

La relación de las variables indicó que, a mayor edad, mayor síntomas depresivos y menor capacidad de agencia de autocuidado; de igual manera se halló que a mayor tiempo de cuidador, mayor síntomas depresivos y menor capacidad de agencia de autocuidado. Así mismo, su ingreso 
mensual mayor se relaciona con menor síntomas depresivo y mayor capacidad de agencia de autocuidado, de igual manera se demostró que a mayor ansiedad, mayores síntomas depresivos; así como mayores síntomas depresivos, menor capacidad de agencia de autocuidado del cuidador (Tabla 3).

Tabla 3

Relación de características del cuidado, ansiedad, depresión y capacidad de agencia de autocuidado en cuidadores informales

\begin{tabular}{|c|c|c|c|c|c|c|}
\hline & 1 & 2 & 3 & 4 & 5 & 6 \\
\hline 1.Edad & 1 & & & & & \\
\hline 2.Tiempo de cuidador & $.458^{* *}$ & 1 & & & & \\
\hline $\begin{array}{l}\text { 3.Ingreso económico } \\
\text { mensual familiar }\end{array}$ & -.191 & $-.243^{*}$ & 1 & & & \\
\hline 4. Ansiedad & $.230^{*}$ & .132 & -.095 & 1 & & \\
\hline 5. Síntomas depresivos & $.436^{* *}$ & $.425^{* *}$ & $-.336^{* *}$ & $.590^{* *}$ & 1 & \\
\hline $\begin{array}{l}\text { 6. Capacidad de agencia } \\
\text { de autocuidado }\end{array}$ & $-.269^{*}$ & $-.203^{*}$ & $.346^{* *}$ & -.030 & $-.323^{* *}$ & 1 \\
\hline
\end{tabular}

${ }^{*} \mathrm{p}<.05,{ }^{* *}<.001$

Finalmente, en el modelo de regresión, se obtuvo que la ansiedad $(\theta=.314)$ y la depresión $(\beta=-.522)$ explicaron un $15 \%$ de la varianza de la capacidad de agencia de autocuidado $(F=8.36$; $p<.01$ ), siendo predictores ambas variables antes referida (ver Tabla 4).

Tabla 4

Modelo de regresión lineal múltiple de los estados afectivos negativos sobre la capacidad de agencia de autocuidado

\begin{tabular}{lcccccccc}
\hline & $B$ & ET & $b$ & $t$ & $F I V$ & $p$ & $F$ & $R^{2}$ \\
\hline & & & & & & .001 & 8.36 & .15 \\
Ansiedad & .203 & .083 & .314 & 2.453 & 1.76 & .016 & & \\
$\begin{array}{l}\text { Síntomas } \\
\text { depresivos }\end{array}$ & -.251 & .061 & -.522 & -4.078 & 1.76 & .000 & & \\
\hline
\end{tabular}

Nota: ET=Error típico, b=Beta, FIV=Factor de inflación de la varianza.

\section{Discusión}

Con base en los resultados las características del cuidador de paciente con enfermedad crónica son similares a investigaciones revisadas en la literatura (Mayoral, 2013; Ruiz et al., 2016) donde indica que el cuidado es otorgado por las mujeres, en etapa de adultez madura, amas de casa y con familiar cercano. Lo anterior es porque el cuidado siempre se ha relacionado con la mujer y aunque existen posibilidades reales de que un hombre sea un cuidador principal, en su mayoría de las ocasiones se elige a una mujer, ya que el cuidado lo establecen como un rol totalmente naturalizado y una obligación moral en este género (Jiménez \& Moya, 2018). 
Por otra parte, en relación con los estados afectivos negativos, la ansiedad estado obtuvo una $M=46.84$ ( $D E=9.83$ ), siendo mayor a lo referido en Perú con una media de 43.23 ( $D E=$ (11.1) y similar al estudio realizado con una media de 46.61 ( $D E=11=29$ ) en Colombia (Guardia, 2013; Méndez et al., 2010). Dichos resultados fueron diferentemente significativos según la ocupación y a las actividades de cuidado que realiza, estos resultados fueron similares al estudiado realizado en Lima, Perú $(p<.05)$, sin embargo, fue diferente a lo reportado en Chiclayo, Perú ( $p>.05$ ) (Guardia, 2013; Segura, 2014). Lo anterior se podría explicar que el ser cuidador con dedicación exclusiva posiblemente favorezca el hecho de que muchos se aíslan de la red social, viven única y exclusivamente para el familiar al que cuidan, transformando la díada cuidador-paciente en un sistema cerrado, así mismo el nivel de dependencia puede ser un factor que aumente los niveles de ansiedad (Cedillo et al., 2015).

La ansiedad se relacionó con la edad $(p<.05)$, pero no con el tiempo de ser cuidador, esto fue similar a lo reportado por la literatura (p>.05) (Méndez et al., 2010; Paz-Rodríguez, 2010), esto pudiera deberse a que pueden existir sistemas de afrontamiento de acuerdo a la edad y tiempo de ser cuidador que pueden influir en el control de la ansiedad y salud psicosocial, así como pueden existir otros factores que sean protectores para la misma.

En cuanto a los síntomas depresivos la media de estudio fue mayor al realizado en Coruña, España con una $\mathrm{x}=22.4$, así como en el realizado en Nueva York, USA con una M=17 y en Santiago, Chile con una $M=16.63$ (Arechabala et al., 2011; Poleshuck et al., 2013; Vázquez et al., 2018). En este sentido se cree que los cuidadores descuidan su salud física y emocional por la sobrecarga del cuidado, ya que se encuentran muy preocupados y al mismo tiempo están obligados a los cuidados que deben de proporcionar a los pacientes la mayor parte del día (Buenfil et al., 2016).

La depresión obtuvo diferencia significativa para la ocupación, estado civil y actividades que realiza el cuidador $(p<.05)$, siendo mayor en las amas de casa y casados, esto fue similar a lo reportado en diversos estudios (Haro, 2014; Paz-Rodríguez, 2010). Una posible explicación va encaminado a la asignación de un rol como cuidador, en algunas ocasiones es por obligación, otras a manera de gratitud o simplemente porqué ningún otro miembro de la familia puede hacerlo, dependiendo de la ocasión puede presentarse una limitación de apoyo social familiar o entendimiento en el cuidado, existiendo la probabilidad de causar una afección en el estado de ánimo del cuidador (Buenfil et al., 2016).

Por otra parte, la agencia de capacidad de autocuidado obtuvo una media en este estudio de 63.82 ( $D E=6.34)$, siendo menor comparado en los estudios realizado en Chile con una media de 70.4 ( $D E=12.5$ ), así como em Colombia con una media de 75.54 ( $D E=10.49$ ) (Hernández et al., 2014; Miranda \& Aravena, 2012). El cuidador informal asume múltiples responsabilidades al momento de cuidado, que limitan sus habilidades de autocuidado basado en su toma de decisiones, responsabilidad del cuidado directo, supervisión, así como acompañamiento y el desarrollo de tareas que cambian en los diferentes escenarios del cuidado que se brinda. Dentro de este proceso en el cuidador confluyen factores internos y externos que afectan de diversas formas su vida, salud y bienestar, pudiendo llegar a un déficit autocuidado (Cantillo-Medina et al., 2018; Ruiz et al., 2019).

Se identificó que la ansiedad y síntomas depresivos influye en un $15 \%$ sobre la capacidad de agencia de autocuidado, siendo ambas variables predictoras. La influencia de la ansiedad y depresión es un peso importante a nivel emocional, lo anterior puedo estar asociado a los diversos roles y responsabilidades en la vida del cuidador, como la familia, el estado ocupacional, 
nivel de ingreso, entre otras. Lo antes referido dificulta el manejo del tiempo propio y las múltiples responsabilidades, así como el sentimiento relacionado con la presión del cuidado del paciente, ocasionando una dificultad para equilibrar las responsabilidades, esto puede abrumar todas las dimensiones de la vida y ocasionando una disminución del autocuidado de la personal (Cedillo et al., 2015; Ruiz et al., 2019).

Con respecto a lo anterior, Landeros et al (2004) refiere que las capacidades de autocuidado pueden estar desarrolladas, pero no operar; es decir, que por alguna razón la persona tiene las habilidades para cuidar de su salud, pero no hace uso de ellas. Esta situación puede estar relacionada con la forma en que las personas perciben su estado de salud, lo que a su vez afecta en sus capacidades de autocuidado. En otras palabras, cuando las personas se perciben sanas, aparentemente no se preocupan por su salud hasta que reconocen o perciben que su salud puede estar en peligro.

Por otra parte, para los profesionales de enfermería es importante la presencia de un cuidador en los pacientes crónicos, sin embargo, el involucramiento en el cuidado puede hacer la diferencia entre la posibilidad de que un paciente finalice un tratamiento y se recupere de un estado agudo o crónico. Es por ello que esta investigación tiene implicaciones en la práctica de cuidado porque el cuidador no solo es hacia el familiar, sino también un cuidado propio, este autocuidado permitirá en la casa o tenga que ser transferido a una unidad de cuidados.

Así mismo la identificación de factores psicológicos que influyen sobre el autocuidado, permitirá que el profesional de enfermería junto con el equipo multidisciplinar, puede promover acciones de intervención a cuidadores informales, con el fin de garantizar un bienestar de la diada cuidador-sujeto de cuidado. Es por ello, se requiere el diseño de proyectos e intervenciones que promuevan el bienestar psicológico, así como disminución de ansiedad y síntomas depresivos del cuidador y su familiar en condición de enfermedad crónica (Hernández et al, 2014).

Entre las limitaciones del estudio se encuentra el reducido tamaño de la muestra, así como la imposibilidad de generalizar los resultados a poblaciones con otras características, debido a que los participantes fueron cuidadores informales de más de dos meses de pacientes adultos mayores con alguna condición crónica. En futuras investigaciones, se recomienda además de aumentar el tamaño de la muestra, seguir estudiando dichas variables ya que se obtuvieron resultados interesantes que pueden influir en el rol cuidador; con lo cual se podrá coadyuvar a la planeación de intervenciones multidisciplinarias que disminuyan los síntomas depresivos y ansiedad en los cuidadores.

En conclusión, los cuidadores de enfermos crónicos en su mayoría estuvieron conformados por mujeres, amas de casa, casados, cuidando un familiar cercano, con necesidades de apoyo en las actividades de la vida diaria, con alto nivel educativo y bajo ingreso económico mensual. Con respecto a los estados afectivos negativos, la media de ansiedad y síntomas depresivos fue mayor a lo referente de literatura, así como la capacidad de agencia de autocuidado. Se encontró una diferencia significativa de la ocupación con la ansiedad, síntomas depresivos y capacidad de agencia de autocuidado, el estado civil solo fue diferente con los síntomas depresivos y los estados afectivos negativos, si fue diferente en las actividades que realiza el cuidador.

Las relaciones señalaron una relación de los síntomas depresivos con la capacidad de agencia de autocuidado, pero no con la ansiedad, sin embargo, el modelo señala que los estados afectivos negativos influyen en un $15 \%$ la capacidad de autocuidado, siendo estos predictores de la misma. 
Dichos resultados servirán para identificar factores que puedan influir en el autocuidado y con el fin de establecer bases para futuras intervenciones de enfermería que puedan disminuir los estados afectivos negativos y mejorar la capacidad de autocuidado en cuidadores de enfermos crónicos.

\section{Referencias}

Arechabala, M. C., Catoni, M. I., Palma, E., \& Barrios, S. (2011). Depresión y autopercepción de la carga del cuidado en pacientes en hemodiálisis y sus cuidadores. Revista Panamericana de Salud Pública/Pan American Journal of Public Health, 30(1), 74-79. Doi: 10.1590/s1020$\underline{49892011000700011}$

Blázquez, F., Soriano-Mas, C., \& Contreras, G. (2012). Afecto positivo y negativo: ¿Una dimensión bipolar o dos dimensiones unipolares independientes? Interdisciplinaria, 29(1), 151-164.

Bojórquez, L., \& Salgado, N. (2009). Características psicométricas de la Escala Center for Epidemiological Studies-depression (CES-D), versiones de 20 y 10 reactivos, en mujeres de una zona rural mexicana. Salud Mental, 32(4), 299-307.

Buenfil, B., Hiijuelos, N., Pineda, J., Salgado, H., \& Pérez, E. (2016). Depresión en cuidadores primarios informales de pacientes con limitación en la actividad. RICS Revista Iberoamericana de Las Ciencias de La Salud, 5(10), 148-173.

Cantillo-Medina, C. P., Ramírez-Perdomo, C. A., \& Perdomo-Romero, A. Y. (2018). Caring ability in family caregivers of people with chronic disease and perceived overload. Ciencia $y$ Enfermería, 24, 1-12. Doi: 10.4067/S0717-95532018000100216

Cedillo, A., Grijalva, M., Santaella, G., Cuevas, M., \& González, A. (2015). Afrontamiento y ansiedad del cuidador primario de pacientes en estado de postración. Revista Médica Instituto Mexicano Seguro Social, 56(3), 362-367.

Cruz-Rivas, J., \& Ostiguin, R. (2011). Cuidador: ¿concepto operativo o preludio teórico? Enfermería Universitaria, 8(1), 49-54. Doi: 10.22201/eneo.23958421e.2010.1.307

Gallegos, E. (1998). Validez y confiabilidad de la versión en español de la escala ASA. Desarrollo Científico de Enfermería, 6(9), 260-264.

García, M. (2018). Nivel de estrés, depresión y ansiedad en cuidadores informales de pacientes con Alzheimer. [Tesis no publicada]. Universidad Miguel de Cervantes.

Gast, H., Denyes, M., Campbell, J., Hartweg, D., Schott-Baer, D., \& Isenberg, M. (1989). Self-care agency: conceptualizations and operationalizations. Advance Nursing Science., 12(1), 2638.

Grove, S. \& Gray, J., (2019). Investigación en enfermería: desarrollo de la práctica de enfermería basada en la evidencia. España. Editorial Elsevier.

Guardia, J. (2013). Ansiedad y afrontamiento en cuidadores de pacientes con esquizofrenia. [Tesis no publicada]. Pontificia Universidad Católica del Perú. 
H. Congreso de la Unión. (2012). Ley general de salud. Ed. SISTA. México .pp 85-102.

Haro, H. (2014). Comparación de la prevalencia de síntomas de depresión y ansiedad en madres que viven dentro y fuera de polígonos con altos índices de violencia social en la ciudad de San Luis Potosí. [Tesis no publicada]. Universidad Autónoma de San Luis Potosí.

Hernández, P., Rodríguez, H., Rojas, J., \& Yacelli, J. (2014). Relación entre nivel de carga del cuidado y capacidad de autocuidado en cuidadores informales de pacientes con enfermedad crónica en el municipio de Tenjo, Cundinamarca en el año 2017. [Tesis no publicada]. Universidad de Ciencias Aplicadas y Ambientales.

Hernández, N; Moreno, C., \& Barragán, J. (2014). Necesidades de cuidado de la díada cuidadorpersona: expectativa de cambio en intervenciones de enfermería. Revista Cuidarte. 5(2) 748-756. Doi: 10.15649/cuidarte.v5i2.87

Jiménez, I., \& Moya, M. (2018). La cuidadora familiar: Sentimiento de obligación naturalizado de la mujer a la hora de cuidar. Enfermería Global, 17(1), 420-433. Doi: 10.6018/eglobal.17.1.292331

Landeros-Olvera, E. A., García-Rojas, J. M., Flores-López, N. C., García-Carlón, A., Vidal-Sánchez, D., \& Vázquez-Pérez, A. (2004). Estimación de las capacidades de autocuidado para hipertensión arterial en una comunidad rural. Rev. Enfermería del Instituto Mexicano del Seguro Social, 12(2), 71-74.

López, Eva (2016). Puesta al día: cuidador informal. Rev Enfermería CyL, 8(1). 71-77.,Disponible en:

http://www.revistaenfermeriacyl.com/index.php/revistaenfermeriacyl/article/viewFile/16 $4 / 144$

Martínez, L. (2018). Ansiedad, depresión y sobrecarga en cuidadores de adultos centenarios. Rev Cubana Salud Pública, 44(4), 61-72.

Mayoral, C. (2013). Los grados olvidados: cuidadores no profesionales de enfermos crónicos dependientes. [Tesis no publicada] Universidad de Zaragoza.

Méndez, L., Giraldo, O., Aguirre, D., \& Lopera, F. (2010). Relación entre ansiedad, depresión, estrés y sobrecarga en cuidadores familiares de personas con demencia tipo alzheimer por mutación e280a en presenilina 1. Revista Chilena de Neuropsicología, 5(2), 137-145.

Mercado, C., Carmen, M., \& Fuentes, C. (2018). Enfermo crónico y cuidador principal. [Tesis no publicada]. Universidad Católica de Murcia.

Miranda, K. E., \& Aravena, V. J. (2012). Sobrecarga, Apoyo Social y Autocuidado en Cuidadores Informales. Ciencia y Enfermería, 18(2), 23-30. Doi: 10.4067/s0717-95532012000200003

Ortiz, L., Franco, C., \& Ortiz, J. (2018). Caracterización sociodemográfica y principales afecciones físicas y psicológicas del cuidador informal. Pensamiento Americano, 11(22), 85-96. Doi: 10.21803/pensam.v11i21-1.265 
Paz-Rodríguez, F. (2010). Predictores de ansiedad y depresión en cuidadores primarios de pacientes neurológicos. Revista Ecuatoriana de Neurología, 19(1-2), 33-41.

Poleshuck, E. L., Talbot, N. L., Moynihan, J. A., Chapman, B. P., \& Heffner, K. L. (2013). Depressive Symptoms, Pain, Chronic Medical Morbidity, and Interleukin-6 among Primary Care Patients. Pain Medicine (United States), 14(5), 686-691. Doi: 10.1111/pme.12089

Retamal-Matus, H., Aguilar, L., Aguilar, M., González, R., \& Valenzano, S. (2015). Factores que influyen en la sobrecarga de los cuidadores de adultos mayores en dos instituciones de la ciudad de Punta Arenas, Chile. Psicogeriatría, 5(3), 119-124.

Ruiz, L., Gordillo, A., \& Galvis, C. (2019). Factores condicionantes básicos en cuidadores informales de pacientes crónicos en el domicilio. Rev Cuidarte, 10(3), 1-15.

Ruiz, M., Martínez, E., López, M., \& Fernández, A. (2016). Evaluación de los estilos de afrontamiento utilizados en cuidadores familiares de enfermos crónicos complejos. European Journal of Health Research: (EJHR), 2(3), 83-93. Doi: 10.1989/ejhr.v2i3.36

Segura, A. (2014). Valoración de la ansiedad y depresión en cuidadores informales del adulto mayor dependiente del Policlínico Chiclayo - Oeste durante julio - diciembre 2012. [Tesis no publicada]. Universidad de San Martin De Porres.

Spielberger, C. D., \& Díaz-Guerrero, R. (2002). IDARE: Inventario de Ansiedad: Rasgo-Estado. Ed el Manual Moderno.

Torres-Avendaño, B., Agudelo-Cifuentes, M. C., Pulgarin-Torres, Á. M., \& Berbesi-Fernández, D. Y. (2018). Factores asociados a la sobrecarga en el cuidador primario. Medellín, 2017. Universidad y Salud, 20(3), 261. Doi: 10.22267/rus.182003.130

Vázquez, F., Otero, P., López, L., Blanco, V., Torres, Á., \& Díaz, O. (2018). La prevención de la depresión en cuidadores a través de multiconferencia telefónica. Clínica y Salud, 29(1), 1420. Doi: $10.5093 /$ clysa2018a2 